\title{
Primary Pulmonary Leiomyosarcoma: Could It Be Cured by Radiation Therapy?
}

\author{
Eun Jung Kim', Yena Kang' ', Do Jin Kim', Dae Sik Hong ${ }^{1}$, Chan Kyu Kim', Eun Suk Ko² \\ Departments of ${ }^{1}$ Internal Medicine and ${ }^{2}$ Pathology, Soonchunhyang University Bucheon Hospital, Bucheon, Korea
}

\begin{abstract}
Primary leiomyosarcoma of lung is extremely rare and often diagnosed as a mass on routine chest radiography. Although advances have been made in treatment protocols, leiomyosarcoma remains one of the more difficult soft tissue sarcomas to treat. Surgical resection is usually curative for small and well-differentiated sarcomas. For poorly differentiated and non-resectable tumors, chemotherapy and radiation therapy are used as neoadejuvant or palliative treatment options. Generally, leiomyosarcomas are known to be resistant to radiation therapy alone. The authors experienced a 68-year-old woman who was diagnosed leiomyosarcoma by routine chest radiography. Although disease progression was observed despite of chemotherapy, radiation therapy reduced the size of tumor. This paper describes the curative potential of radiation therapy for primary pulmonary leiomyosarcomas through a case report and literature review.
\end{abstract}

Keywords: Leiomyosarcoma; Radiotherapy; Chemotherapy

\section{INTRODUCTION}

Pulmonary sarcomas of lung are rare, accounting less than $0.5 \%$ of all malignant lung tumors [1]. The most common types of primary sarcomas of lung were fibrosarcomas, leiomyosarcomas, and hemangiopericytomas [2]. Primary pulmonary leiomyosarcomas (PPL) are extremely rare and often diagnosed as a mass on routine chest radiography. It can arise from the smooth muscle cells of pulmonary parenchyma, bronchi, bronchioles, or bronchial arterioles in order to decrease frequency [3]. Although advances have been made in treatment protocols, leiomyosarcoma remains one of the more difficult soft-tissue sarcomas to treat. Applicable therapeutic methods include surgery, radiation therapy, and chemotherapy. Surgical resection, if complete, which is usually curative for small and well-differentiated sarcomas. Poorly differentiated and non-resectable tumors can be treated with palliative radiotherapy or chemotherapy, but the results are not satisfactory [4]. In this report, authors tried radiation therapy for a patient who had multiple poor prognostic factors and had no response to chemotherapy. We recommend multidisciplinary treatment planning and radiation therapy can be an option for PPL in selective cases.

\section{CASE REPORT}

A 68-year-old non-smoker woman with a known history of hypertension and rheumatic arthritis was referred to Sooncheonhyang Bucheon Hospital for the evaluation of the solitary pulmonary nodule (SPN) on chest radiograph. She complained non-productive cough and weight loss. Chest computed tomography (CT) showed $1.7 \times 1.2-\mathrm{cm}$-sized nodule in anterior segment of right upper lobe (Fig. 1A). Bronchoscopy and percutaneous transthoracic needle biopsy (PTNB) was done. Biopsy revealed patchy smooth muscle proliferation and malignancy could not be ruled out. Surgical resection for the SPN was recommended, but the patient refused. She revisited our clinic for chest pain, cough, and sputum 2 years later. Previous mass size was much increased in chest CT $(6.5 \times 4.0 \mathrm{~cm})$ (Fig. 1B). PTNB was performed again. The biopsy showed spindle cell proliferation with nuclear atypia and increased mitosis $(>10 / 10$ high power field) (Fig. 2A). Immunohistochemical stains presented positive for actin \& desmin and negative for CK-pan, CD34, CD117, and S-100, compatible with leiomyosarcoma (Fig. 2B). Because the primary leiomyosarcoma of lung was extremely rare, additional positron emission tomography (PET)-CT scan and gynecologic
Correspondence to: Chan Kyu Kim

Division of Hematology \& Oncology, Department of Internal Medicine, Soonchunhyang University Bucheon Hospital,

170 Jomaru-ro, Wonmi-gu, Bucheon 14584, Korea

Tel: +82-32-621-5186, Fax: +82-32-621-5016, E-mail: md53097@schmc.ac.kr

Received: Sep. 26, 2015 / Accepted after revision: Oct. 21, 2015
(C) 2015 Soonchunhyang Medical Research Institute This is an Open Access article distributed under the terms of the Creative Commons Attribution Non-Commercial License (http://creativecommons.org/licenses/by-nc/3.0/). 

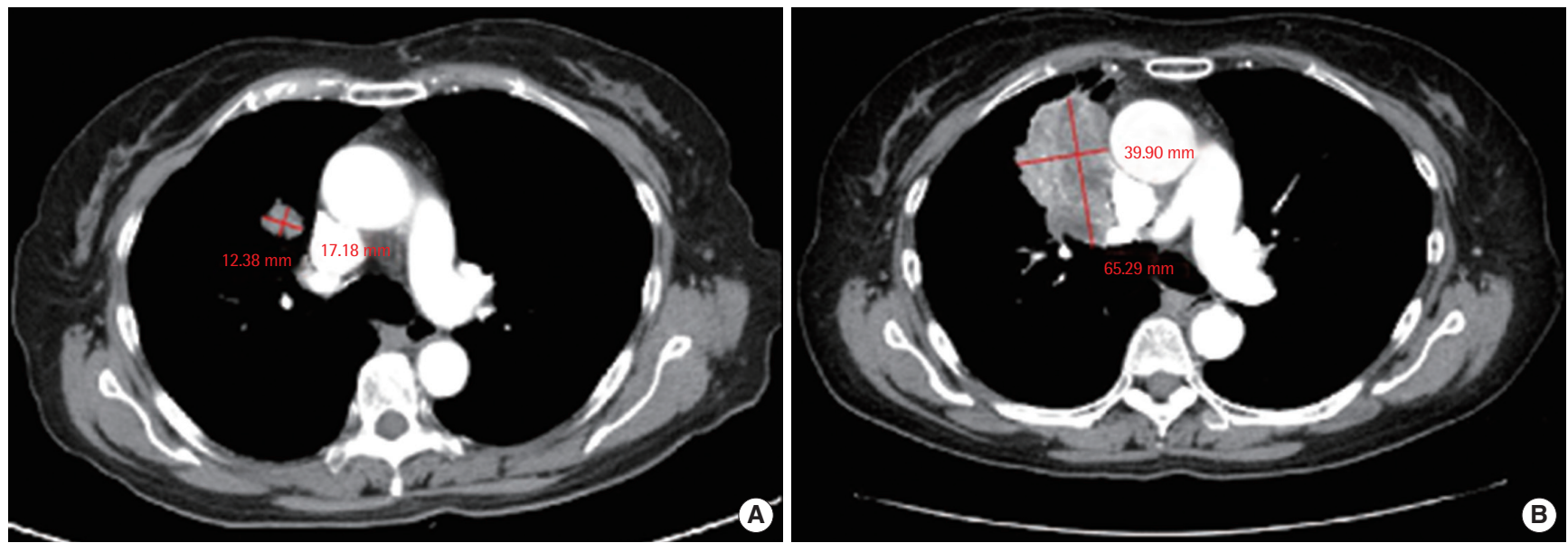

Fig. 1. (A) Initial mass presented as solitary pulmonary nodule and (B) increased mass size 2 years later.
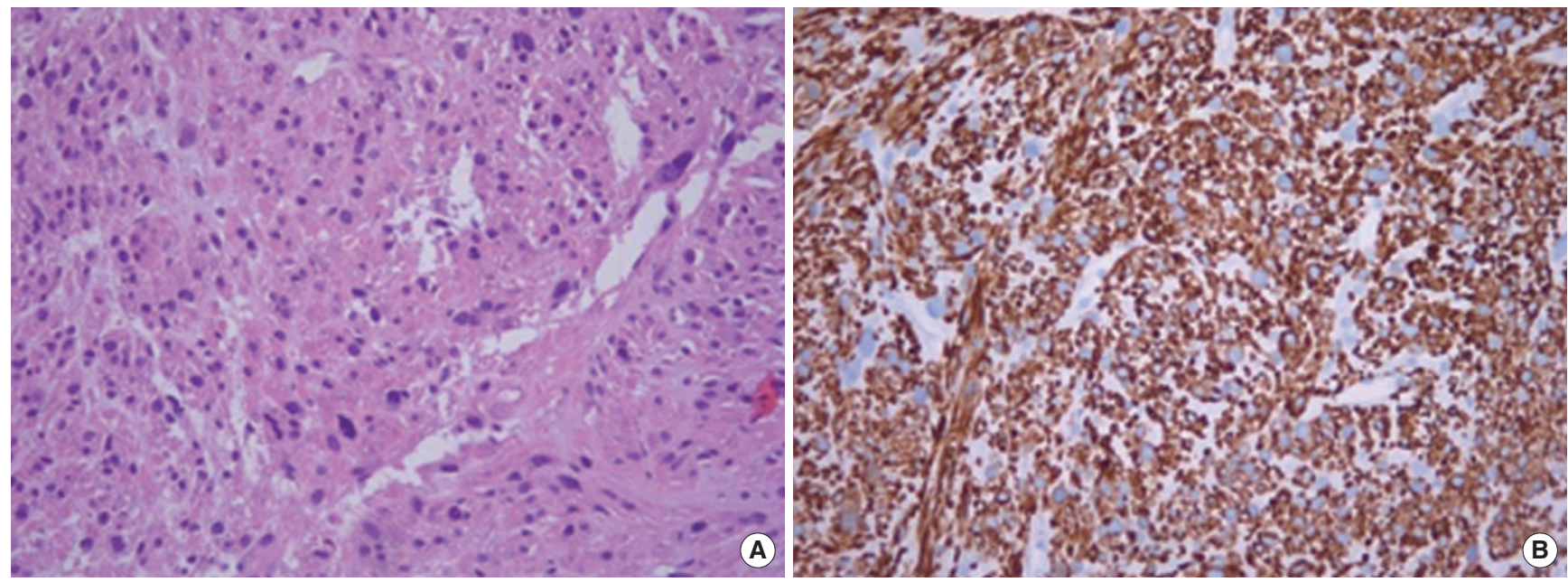

Fig. 2. (A) Percutaneous transthoracic needle biopsy shows fascicular arrangement of spindle cells with eosinophilic cytoplasm and blunted end nuclei. Occasional mitoses are also noted $(H \& E, \times 200)$ and $(B)$ immunohistochemical stain for desmin reveals diffuse strong positivity in the tumor cells. Findings are consistent with leiomyosarcoma (immunohistochemistry, $\times 200$ ).

ultrasonography were done. There was no evidence of other primary focus except pulmonary mass (Fig. 3A).

She was diagnosed as primary pulmonary leiomyosarcoma. Considering cancer size, location, and lymph node uptake at PETCT, complete surgical resection was thought to be impossible by chest physicians. She was treated with adriamycin and ifosfamide combination chemotherapy to reduce the size of mass. Tumor response evaluation was done after 2 cycles of chemotherapy. Follow-up chest CT showed aggravation of the tumor (Fig. 3B). Multidisciplinary cancer care team had to decide next treatment option. Radiation oncologists recommended radiation therapy. Thus, radiation (5,400 cGy/30 fractions) was applied for 2 months. Followup chest CT was done after 2 and 6 months from the end of radia- tion therapy. The size of tumor was decreased gradually, measured $2.9 \times 1.4 \mathrm{~cm}$ finally (Fig. 3C) with conservative treatment. Authors expected that radiation therapy may control the tumor as stable state. However, 5 months later, chest CT showed the mass with more increased size $(2.8 \times 2.6 \mathrm{~cm})$ (Fig. $4 \mathrm{~A})$. Our multidisciplinary cancer care team had to decide another treatment option. Highdose ifosfamide may be an option for patients who already received standard-dose ifosfamide. Trabectedin and dacarbazine are 2nd-line options which proved effective in leiomyosarcoma [5]. But the patient seemed not to bear high-dose chemotherapy and second-line options were not available. Since the first radiation therapy had good response, tomotherapy (6,900 cGy/30 fractions) was applied for the next 6 weeks. Follow-up chest CT was done af- 
Kim EJ, et al. • The Curative Potential of Radiation Therapy for Primary Pulmonary Leiomyosarcoma
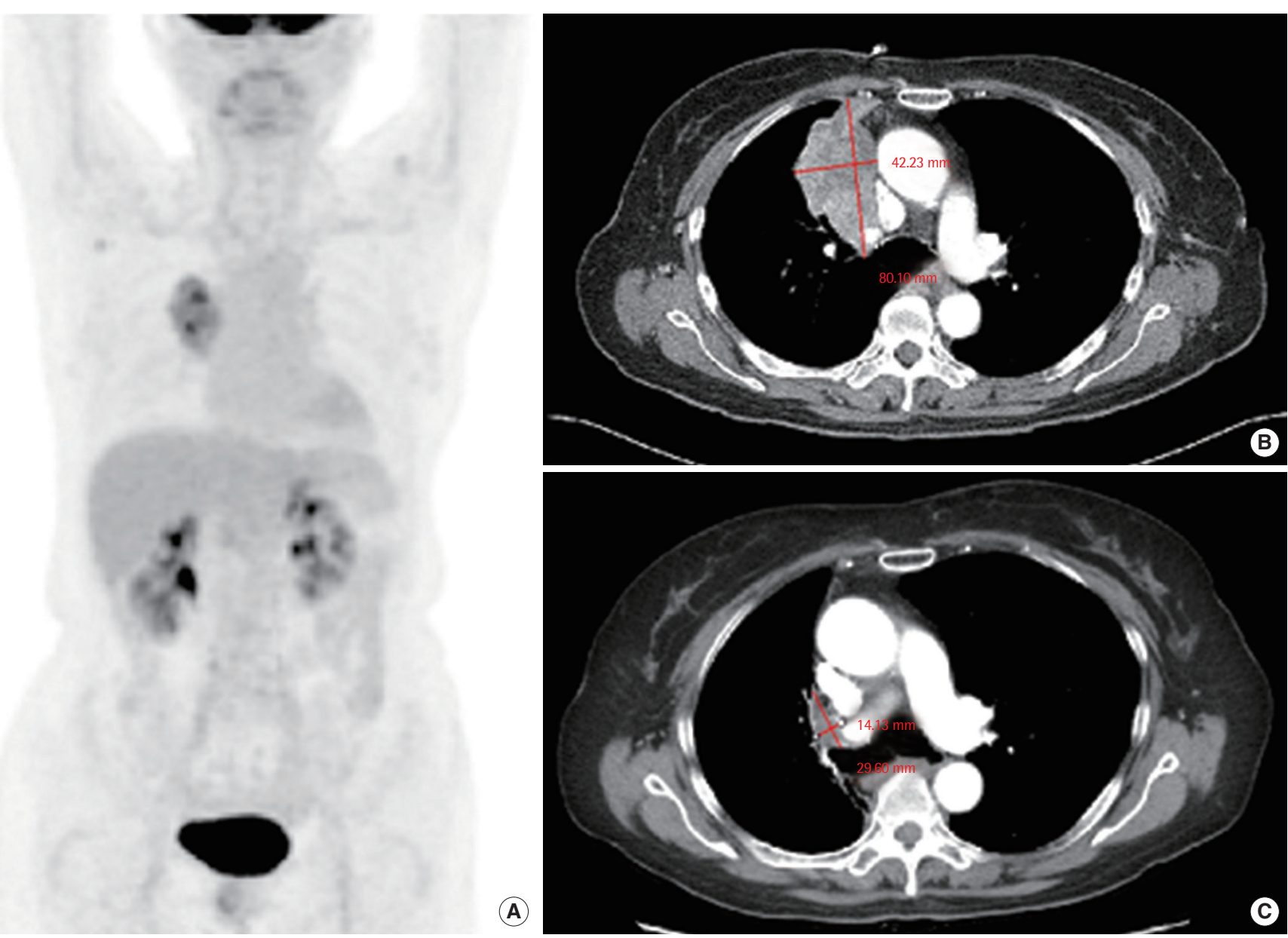

(A)

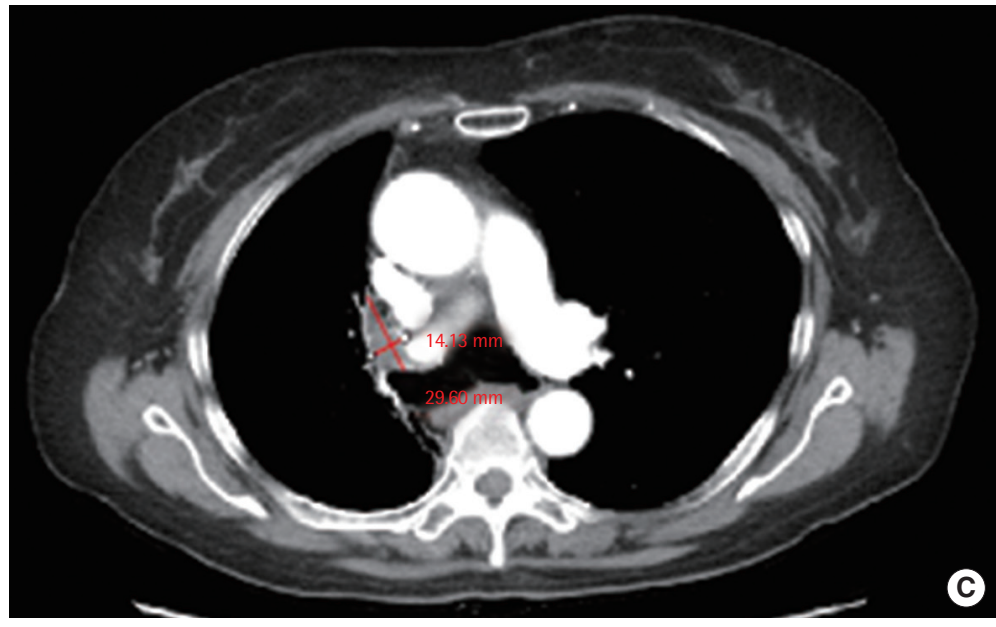

Fig. 3. (A) Positron emission tomography-computed tomography scan shows no evidence of leiomyosarcoma except pulmonary mass. (B) The tumor size aggravated after 2 cycles of chemotherapy with adriamycin and ifosfamide. (C) Radiation was applied for the next 2 months and the tumor size decreased significantly 6 months after radiation.
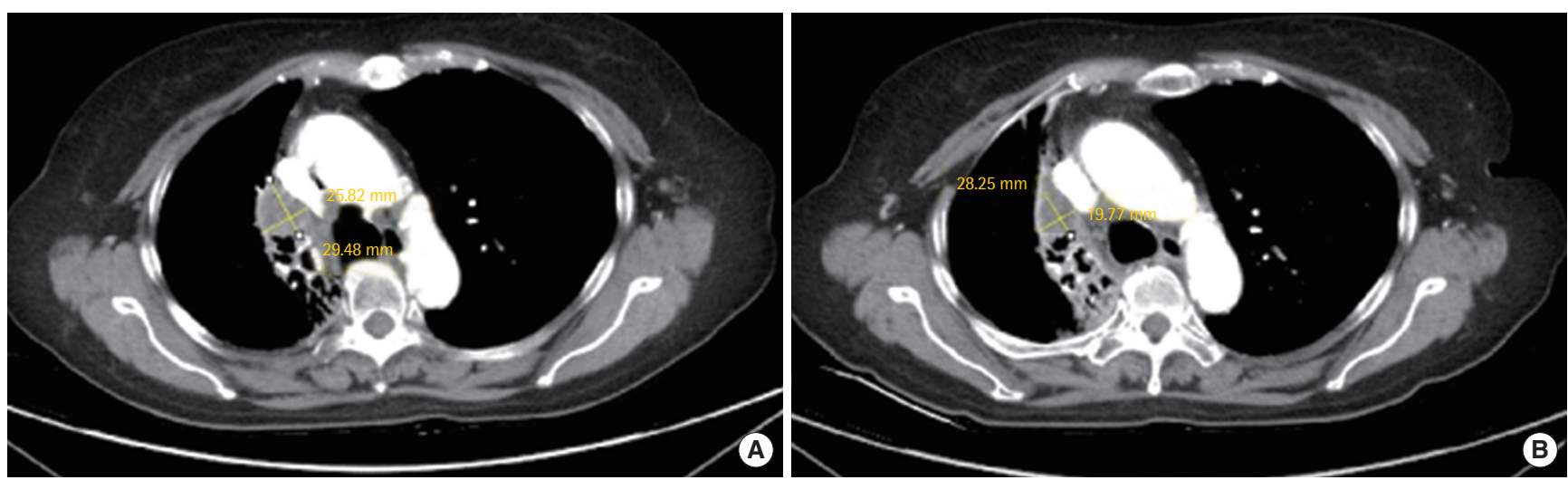

Fig. 4. (A) Five months later, the mass grown up again. (B) Follow-up chest computed tomography showed decreased tumor size after additional tomotherapy for 6 weeks.

ter 6 weeks from the end of the radiation and the size of tumor was decreased again $(2.8 \times 2.0 \mathrm{~cm})$ (Fig. 4B). However, she suffered from right pneumonitis and she expired by sepsis 1 month later.

\section{DISCUSSION}

Pulmonary sarcomas of lung are rare, accounting less than $0.5 \%$ of all malignant lung tumors [1]. It can arise from the smooth mus- 
cle cells of pulmonary parenchyma, bronchi, bronchioles, or bronchial arterioles in order to decrease frequency [2]. Patients may present with symptoms similar to those seen in other primary pulmonary tumors, including cough, dyspnea, hemoptysis, sputum, chest or back pain, and weight loss. Diagnosis of primary pulmonary leiomyosarcoma needs multiple core needle biopsies or an excisional biopsy [5]. After tissue biopsy, preoperative staging generally includes CT scan of the lung and magnetic resonance imaging of the primary lesion to determine whether metastases have occurred [6]. Surgical resection is usually curative for small and welldifferentiated sarcomas. Surgery through wide excision may be coupled with adjuvant radiation therapy and adjuvant chemotherapy as standard treatment. If complete, median survival is 48 months and the 5-year survival rate ranges from 29\% to 40\% [7]. Unfortunately, only one-third of the cases can be treated surgically due to patient factors or the extension of the tumor [8]. In poorly differentiated and non-resectable tumors, chemotherapy and/or radiotherapy are treatment options. Regional lymph node metastases are rare and constitute an adverse prognostic factor. Metastasis is uncommon and typically occurs late in the disease process [9].

In this case, patient had poorly differentiated, non-resectable tumor and cannot be ruled out lymph node metastasis. We tried chemotherapy first with adriamycin and ifosfamide combination, but tumor had been aggravated. After radiation therapy $(5,400$ cGy/30 fractions), the tumor size decreased gradually and authors expected that radiation therapy may control the tumor stable state. But after 11 months from the radiation therapy, the tumor size was increased and after 6 weeks from the 2nd radiation therapy, tumor size decreased again. Our team decided to try additional radiation therapy to control the tumor.

In a recent study, Navarria et al. [10] reported the role of stereotactic body radiation therapy in 28 patients with lung metastasis from primary soft tissue sarcoma. Overall survival at 2 and 5 years after radiation therapy was improved $(96.2 \%$ and $60.5 \%$, respectively) and no severe toxicity was recorded. Further studies are need to elucidate the potential role of definitive radiation therapy for unresectable locally advanced primary pulmonary leiomyosarcoma.

In summary, we recommend radiation therapy in primary pulmonary leiomyosacoma as a potent curative option in selected cases. Before treatment, multidisciplinary treatment planning should be made to decide radiation doses, schedules, and devices. Further study is needed for clinical guidelines in radiation therapy for primary pulmonary leiomyosarcoma.

\section{REFERENCES}

1. Attanoos RL, Appleton MA, Gibbs AR. Primary sarcomas of the lung: a clinicopathological and immunohistochemical study of 14 cases. Histopathology 1996;29:29-36.

2. Nascimento AG, Unni KK, Bernatz PE. Sarcomas of the lung. Mayo Clin Proc 1982;57:355-9.

3. Shoji F, Yoshino I, Takeshita M, Sumiyoshi S, Sueishi K, Maehara Y. Pulmonary leiomyosarcoma presenting as a pancoast tumor. Pathol Res Pract 2007;203:745-8.

4. Arnold LM 3rd, Burman SD, O-Yurvati AH. Diagnosis and management of primary pulmonary leiomyosarcoma. J Am Osteopath Assoc 2010; 110:244-6.

5. Casali PG, Jost L, Sleijfer S, Verweij J, Blay JY; ESMO Guidelines Working Group. Soft tissue sarcomas: ESMO clinical recommendations for diagnosis, treatment and follow-up. Ann Oncol 2008;19 Suppl 2:ii89-93.

6. Hayashi T, Tagawa T, Ashizawa K, Abe K, Kinoshita N, Tsutsui S, et al. Contrast-enhanced dynamic magnetic resonance imaging of primary pulmonary leiomyosarcoma. Tohoku J Exp Med 2006;210:263-7.

7. Naik MJ, Lim CH, Agasthian T. Primary pulmonary leiomyosarcoma: a rare presentation. Asian Cardiovasc Thorac Ann 2000;8:67-9.

8. Luthra M, Khan H, Suhail MF, Avadhani V. Primary pulmonary leiomyosarcoma: a case report. Arch Bronconeumol 2012;48:476-8.

9. Ramanathan. Primary leiomyosarcoma of the lung. Thorax 1974;29: 482-9.

10. Navarria P, Ascolese AM, Cozzi L, Tomatis S, D’Agostino GR, De Rose F, et al. Stereotactic body radiation therapy for lung metastases from soft tissue sarcoma. Eur J Cancer 2015;51:668-74. 